\title{
Bladder utility symptom scale: a new patient reported outcome measure for health-related quality of life in bladder cancer
}

\author{
Bhaskar K. Somani \\ Department of Urology, University Hospital Southampton NHS Trust, Southampton, UK \\ Correspondence to: Bhaskar K. Somani. Department of Urology, University Hospital Southampton NHS Trust, SO16 6YD, Southampton, UK. \\ Email: bhaskarsomani@yahoo.com. \\ Provenance: This is a Guest Editorial commissioned by Section Editor Xiao Li (Department of Urology, Jiangsu Cancer Hospital \& Jiangsu Institute \\ of Cancer Research \& Nanjing Medical University Affiliated Cancer Hospital, Nanjing, China). \\ Comment on: Perlis N, Krahn MD, Boehme KE, et al. The Bladder Utility Symptom Scale (BUSS): A Novel Patient-Reported Outcome Instrument \\ in Bladder Cancer. J Urol 2018;200:283-91.
}

Submitted Jul 09, 2018. Accepted for publication Jul 17, 2018.

doi: $10.21037 /$ tau.2018.07.10

View this article at: http://dx.doi.org/10.21037/tau.2018.07.10

Quality of life (QoL) is a subjective experience and measuring it can be challenging given the complexities in the nature of presentation and management of bladder cancer (1-4). However, QoL must be determined by the patients rather than by clinicians and it is important to consider how the disease affects them. This might depend on the severity or nature of their disease and possibly the treatment as perceived by the patients. Apart from symptom related aspect of the disease, psychological issues must also be considered (2). Although a wide range of QoL tools exist, the key is for patients themselves to assess it, and not for the surgical or oncological teams to try and assume what their QoL might be. The aim of any treatment is for a given patient to feel and function normally and QoL measurement is one of the aspects in this regard. Over the last 3 decades there have been a number of generic and bladder cancer specific QoL tools (2-4).

Generic and disease specific measure have its own strengths and limitations. While generic instruments allow simultaneous measurement of various QoL domains, these may not be of interest to the assessor or the patient. Similarly, although they allow for comparison of different bladder cancer patients across different populations but may not capture small but clinically important changes. Disease specific measure on the other hand allows to focus on clinically relevant areas and can be specific to cancer or organ function or the population that is being studied, although they may not be able to compare QoL of different disease conditions. Hence, the clinical teams should identify the domains of interest prior to choosing the QoL instrument.

Bladder utility symptom scale (BUSS) is a new patient reported outcome (PRO) measure. It has been developed to measure health related quality of life (HRQoL) in patients with non-invasive, invasive and metastatic bladder cancer. It has a 10 -item questionnaire and these 10 questions explore the most important areas of relevance to patients. With involvement of patient and clinician focus groups, BUSS is suitable for all bladder cancer patients incorporating generic and bladder cancer specific domains.

Previous studies on QoL show a wide variability in the way it was measured with different validated and nonvalidated questionnaires used. Although the last few years have seen an increase in the number of studies using validated questionnaires, majority are still retrospective in nature measuring only the post-operative QoL $(5,6)$.

The emphasis of bladder cancer treatment is no longer limited to the disease itself but has shifted to ensure patients enjoy a good QoL. For assessment of QoL, the instrument should have good psychometric properties such as reliability, validity and responsiveness. The aims should be clearly defined, and the measurement should be relevant and appropriate, incorporating cultural values in it. There are several generic and disease specific QoL tools available for bladder cancer patients. Being multidimensional, in addition to health these also cover emotional, physical, social and possibly spiritual aspects of a patient giving a 
perspective and insight into their own illness.

BUSS was developed with rigorous methodology (over 3 studies) including pilot testing followed by testing for validity and reliability and subsequently for its generalizability and external validation. During the development the other 4 instruments used were the generic instruments (EQ-5D-3L and SF-36) and the disease specific instruments (FACT-BL and BCI). Patients from various bladder cancer backgrounds were included (surgery, radiotherapy and chemotherapy). Each of the questions developed were designed to check on how that domain affected their daily life, which was a central issue was raised by previous patient groups. With a short time to administer (10 minutes), BUSS can be administered with ease in clinics.

While BUSS measures global HRQoL in any patient with bladder cancer, it cannot be used to measure QoL in patients with non-cancer related urinary diversion (7). Similarly, longitudinal measurements on response to treatment has not been measured or reported yet. Hopefully, this will be done in future studies along with its uptake and utility in trials and comparative effectiveness research. The QoL measurement should be done prior to and post-intervention, analysed by independent third party to minimise bias associated with the modality of treatment chosen. Perhaps a key area in the future would be decision aids to help patients choose the right treatment based on clinical nomograms and oncological outcomes taking into account "domains" or areas of QoL most important to them (8). This could be achieved by QoL interviews or using tools such as SEIQoL-DW (4). Pre-treatment, it would allow areas most important to patients which could potentially be incorporated in these decision-making tools and post-treatment, it could help analyse the outcomes and side-effects from the treatment received. For wider uptake including people who are less educated, these would need to use common words, short sentences giving visual aids or examples where appropriate.

In future, studies using BUSS should focus on randomised controlled trials and if this is not feasible, at least attempt to collect prospective data at baseline and then at multiple time points after treatment. It seems that with BUSS, the future in measuring QoL in patients with

Cite this article as: Somani BK. Bladder utility symptom scale: a new patient reported outcome measure for healthrelated quality of life in bladder cancer. Transl Androl Urol 2018;7(4):740-741. doi: 10.21037/tau.2018.07.10 bladder cancer looks promising.

\section{Acknowledgements}

None.

\section{Footnote}

Conflicts of Interest: The author has no conflicts of interest to declare.

\section{References}

1. Perlis N, Krahn MD, Boehme KE, et al. The Bladder Utility Symptom Scale: A Novel Patient Reported Outcome Instrument for Bladder Cancer. J Urol 2018;200:283-91.

2. Somani BK, MacLennan SJ, N'Dow J, et al. Quality of Life With Urinary Diversion. Eur Urol Suppl 2010;9:763-71.

3. Somani BK, Nabi G, Wong S, et al. How close are we to knowing whether orthotopic bladder replacement surgery is the new gold standard?--evidence from a systematic review update. Urology 2009;74:1331-9.

4. Somani BK, Gimlin D, Fayers P, et al. Quality of life and body image for bladder cancer patients undergoing radical cystectomy and urinary diversion--a prospective cohort study with a systematic review of literature. Urology 2009;74:1138-43.

5. Kim SP, Shah ND, Weight CJ, et al. Population-based trends in urinary diversion among patients undergoing radical cystectomy for bladder cancer. BJU Int 2013;112:478-84.

6. Gore JL, Litwin MS, Urologic Quality of care in bladder cancer: trends in urinary diversion following radical cystectomy. World J Urol 2009;27:45-50.

7. Chakravarti A, Ganta S, Somani B, et al. Caecocystoplasty for intractable interstitial cystitis - long term result. Eur Urol 2004;46:114-7.

8. O'Connor AM, Bennett CL, Stacey D, et al. Decision aids for people facing health treatment or screening decisions. Cochrane Database Syst Rev 2009;(3):CD001431. 